\title{
Magnetic catalysis of a charged Bose-Einstein condensate
}

\author{
Alejandro Ayala, ${ }^{1}$ M. Loewe,${ }^{2,3}$ Juan Cristobal Rojas, ${ }^{4}$ C. Villavicencio ${ }^{5,6}$ \\ ${ }^{1}$ Instituto de Ciencias Nucleares, Universidad Nacional Autónoma de México, \\ Apartado Postal 70-543, México Distrito Federal 04510, Mexico \\ ${ }^{2}$ Facultad de Física, Pontificia Universidad Católica de Chile, Casilla 306, Santiago 22, Chile \\ ${ }^{3}$ Centre for Theoretical and Mathematical Physics, \\ University of Cape Town, Rondebosch 7r00, South Africa \\ 4 Departamento de Física, Universidad Católica del Norte, Casilla 1280, Antofagasta, Chile \\ ${ }^{5}$ Departamento de Física and Centro-Científico-Tecnológico de Valparaíso, \\ Universidad Técnica Federico Santa María, Casilla 110-V, Valparaiso, Chile \\ ${ }^{6}$ Universidad Diego Portales, Casilla 298-V, Santiago, Chile
}

\begin{abstract}
We study the condensation phenomenon for a system of charged bosons in the presence of an external magnetic field. We show that condensation happens for a definite critical temperature instead of through a diffuse phase transition. The essential ingredient, overlooked in previous analyses and accounted for in this work, is the treatment of the plasma screening effects by means of resummation. We compute the critical temperature, for the case in which the condensate is made of charged pions and for typical densities found in compact astrophysical objects, for small and large values of the magnetic field. We show that the magnetic field catalyzes the onset of condensation at very small and at large values of the magnetic field, and that for intermediate values the critical temperature for condensation is lower than for the zero magnetic field case.
\end{abstract}

PACS numbers: 11.10.Wx, 67.85.Jk, 26.60.Dd

Keywords: Bose-Einstein condensate, Charged scalar field, Chemical potential, Uniform magnetic field

\section{INTRODUCTION}

The possibility that a charged pion condensate may occur in the interior of neutron stars has been repeatedly examined in the past. This possibility is raised by the large isospin imbalance between neutrons and protons which favors reactions that make neutrons decay into negative pions under appropriate conditions. The equilibrium thermodynamic conditions obeyed by a pion condensed state in dense neutron matter and in neutron stars have been discussed long ago. In particular, Ref. [1] studies the criteria for the appearance of pion condensation in neutron matter in terms of the pion Green's function (for a general review on the physics of neutron stars see Ref. [2]). The occurrence of a charged boson condensed phase without magnetic fields has also been extensively discussed in the literature. Refs. 3] [6] study in-medium processes introducing an isospin chemical potential $\mu_{I}$ at zero temperature in both phases $\left(\left|\mu_{I}\right| \gtrless m_{\pi}\right.$, where $m_{\pi}$ is the pion mass), analyzing the formation of a charged pion condensed phase. This phenomenon was discussed in electrically neutral dense quark matter in Refs. 79]. Finite temperature corrections, in the frame of chiral perturbation theory, have been considered in Ref. [10], extending the discussion also to other condensates, like the chiral condensate or the axial-isospin charge density condensate, in Ref. [11].

The situation becomes even more interesting when considering that neutron stars possess large magnetic fields whose effects should also be included when studying the condensation conditions. Recently, in Ref. [12] the role played by the coupling of $\pi^{0}$ to a magnetic field via the triangle anomaly has been considered, showing the emer- gence of a $\pi^{0}$ domain wall for values of the magnetic field strength $B$ larger than a certain critical value. This could also happen for $\left(\eta, \eta^{\prime}\right)$ states when $B \sim 10^{7}-10^{19} \mathrm{G}$.

Magnetic fields can also play an important role in the dynamics of systems where charged pions are copiously produced, such as relativistic heavy-ion (RHI) collisions. Recently, the importance of large magnetic fields for the evolution of QCD matter produced in noncentral RHI collisions has been discussed in Ref. [13] as well as their influence on the phase structure of QCD, with emphasis on the chiral symmetry restoration and deconfining transitions. In Ref. [14], a discussion of the effective potential in the framework of the linear sigma model, coupled to quarks and/or Polyakov loop, suggests a richer structure of the strong interactions like, for example, a possible splitting between chiral symmetry restoration and deconfinement in the presence of magnetic fields. The influence of the external magnetic field on the formation of CPodd domains in RHI collisions has also been discussed in Ref. [15]. A decrease in the confining critical temperature was found in [16], where a hadron-quark transition was studied within the MIT bag model. With the above ingredients put together, the theoretical study of a charged boson condensate with a finite chemical potential in the presence of magnetic fields becomes even more relevant. Although this is an old problem, the results from several approaches vary in their conclusions. For instance, it was long ago argued that a nonrelativistic Bose-Einstein gas of charged particles does not condense in the presence of a magnetic field, regardless of how weak the field may be [17]. This result motivated the search for conditions where condensation could take place with magnetic fields, in particular to study whether this could happen extending the number of spatial dimensions [18 20]. In the 
nonrelativistic case, treating the dimensionality $d$ of the system as a continuous variable, it was shown in Ref. [18] that condensation can happen only for $d>4$. For pairs of bosons or fermions and in the relativistic case, it was shown in Ref. [19] that for the case when $d$ is taken as an integer, condensation happens for odd $d \geq 5$. A similar conclusion was reached in Ref. [20], although these authors also realized that the lowest Landau level can play the role of the ground state to accommodate a large charge density in the $d=3$ case.

The common feature of all of the above-mentioned analyses is the definition of the condensation condition which is taken as the equality of the chemical potential and the ground state energy. However, in the presence of a magnetic field, this condition leads to a divergence of the particle density for that state. Indeed, since for a constant magnetic field the energy levels separate into transverse and longitudinal (with respect to the magnetic field direction) and the former are described in terms of discrete energy levels, the divergence of the Bose-Einstein distribution when the chemical potential is equal to the lowest energy level can only be cured in a larger than $d=4$ number of spatial dimensions.

The implications of this condition were recognized in Ref. 21] where it was argued that when the temperature $T$ is much lower than $e B$ one can already consider that the system occupies only the lowest Landau level. This means that the value for the chemical potential to compute the ground state density does not need to be equal to the lowest energy. In this picture the occupation of this state occurs without the need of having a critical temperature, that is, the system undergoes a diffuse phase transition.

Nevertheless one can argue that if in the absence of a magnetic field the system is already in the condensed phase with a macroscopic fraction of the population occupying the lowest energy level, a slow turning on of the magnetic field should not lead to the instantaneous destruction of the condensate. Put in equivalent terms, the onset of condensation for small magnetic fields should be a phenomenon that takes place at a given critical temperature $T_{c}$ since it does so in the limit of a vanishing magnetic field and the presence of a small one cannot drastically change the picture. To implement this idea, one should keep in mind that the chemical potential is not a number that can arbitrarily be set to take a specific value but rather, a function of the thermodynamic variables such as temperature and density. Its value should be determined by demanding that the ground state is populated by a finite charge density. The missing ingredient that bridges the gap in the analysis is to consider the plasma screening effects, which are of course needed since we are dealing with infrared phenomena where the effective mass is small or may even vanish.

In this work we study the conditions for the onset of a condensed phase for a charged boson system, in the presence of an external magnetic field. To mimic the situation where there is an isospin imbalance, we introduce a finite chemical potential $\mu$. For the description, we resort to model the boson system in terms of a theory of a charged scalar with quartic self-interactions. We show that for small and large values of the magnetic field, the system presents the magnetic catalysis phenomenon [22]; that is, that the formation of the condensate is favored by the presence of the magnetic field. This phenomenon has also been found in the context of the Nambu-JonaLasino model at $T=0$ 23] and in $(2+1)$ dimensions both at $T=0$ and $T \neq 0$ [24], where it was shown that even the presence of an arbitrary small magnetic field breaks the chiral invariance of the models. A main result of our work is to show that when including the plasma screening effects, there is a well-defined critical temperature associated with the onset of condensation. A similar calculation, using optimized perturbation theory, albeit without the introduction of a chemical potential, was done in Ref. [25]. The authors found that the phase transition is always second order and the magnetic catalysis phenomenon is present for all values of the magnetic field. They also found that the critical temperature increases with increasing values of the magnetic field.

The work is organized as follows: In Sec. [II we find the lowest energy state where condensation happens and define the order parameter for the transition. In Sec. III we compute the one-loop corrections to the grand potential and set up the discussion for the onset of the condensation phenomenon in terms of the existence of a large but finite charge density in the ground state. In Sec. IV we revisit the description of the onset of condensation when corrections from interactions are accounted for. We take the limit $B \rightarrow 0$ and point out the need to include plasma screening effects by means of resummation, even in this case. In Sec $\nabla$ we explicitly compute the resummed selfenergy for finite $B$ in the low temperature approximation in the limits of small and large magnetic fields. This selfenergy is then used in Sec. VI to compute the critical temperature for condensation when the charged bosons are taken as pions, for typical densities in compact astrophysical objects such as neutron stars. We finally summarize and conclude in Sec. VII.

\section{ORDER PARAMETER}

We want first to define the order parameter that describes the condensation transition. This is a delicate task since (as we will show) in the presence of a magnetic field, the condensate does not correspond to a spatially uniform state. Let us start by introducing the Lagrangian representing a charged scalar field $\phi$ with finite chemical potential $\mu$ interacting with a uniform external magnetic field $\boldsymbol{B}$ oriented in the $z$ direction. Working in the symmetric gauge, the vector potential corresponding to the given magnetic field can be written as

$$
\boldsymbol{A}=\frac{1}{2} \boldsymbol{B} \times \boldsymbol{r}
$$


In Euclidean space and after introducing the magnetic field by means of the minimal substitution we get

$$
\begin{aligned}
\mathcal{L}_{\mathrm{E}} & =\left(\partial_{\tau}+\mu\right) \phi^{*}\left(\partial_{\tau}-\mu\right) \phi+|(\boldsymbol{\nabla}-i q \boldsymbol{A}) \phi|^{2} \\
& +m^{2}|\phi|^{2}+\frac{\lambda}{4}|\phi|^{4}+\delta \mathcal{L},
\end{aligned}
$$

where $\delta \mathcal{L}$ contains the ultraviolet counterterms and $q$ is the (positive) charge associated to the field $\phi$. We want to describe the situation where for a given value of the chemical potential, the system develops a superfluid phase characterized by a boson condensate described by a classical field $\phi_{c}$; namely, that the field can be expressed as

$$
\phi=\frac{1}{\sqrt{2}} \phi_{c}+\tilde{\phi}
$$

where $\tilde{\phi}$ is the quantum field, as referred from the real classical ground state $\phi_{c}$. In the presence of an external magnetic field, the classical equation of motion does not allow a constant value for $\phi_{c}$ [26] and thus $\phi_{c}$ cannot simply be taken as the order parameter for the transition. In order to see how one can proceed in such a situation, let us find the ground state. Using the Lagrangian in Eq. (2) we obtain the classical action

$$
\begin{aligned}
\Gamma_{c} & =\beta \int d^{3} x\left\{\frac{1}{2} \phi_{c}\left(-\nabla^{2}+q^{2} \boldsymbol{A}^{2}+m^{2}-\mu^{2}\right) \phi_{c}\right. \\
& \left.+\frac{\lambda}{16} \phi_{c}^{4}\right\}
\end{aligned}
$$

where we have discarded a surface term after integration by parts and $\beta=1 / T$. When the space boundary is not strictly taken at infinity, the surface term does not vanish; however, given the form of the classical solution [see Eq. (77) below] the boundary contribution can be neglected for a sufficiently large volume. We first look for a solution for the free case $(\lambda=0)$; thus, the eigenvalue problem for the classical equation of motion becomes

$$
\left[-\nabla^{2}+(q B)^{2}\left(x^{2}+y^{2}\right) / 4+m^{2}-\mu^{2}\right] \phi_{c}=\mathcal{E}^{2} \phi_{c}
$$

which is recognized as a two-dimensional harmonic oscillator whose eigenvalues are given by

$$
\mathcal{E}_{l}^{2}\left(p_{z}\right)=p_{z}^{2}+m^{2}+(2 l+1) q B-\mu^{2},
$$

where $l \geq 0$ labels the Landau level. Let us specialize to the lowest energy state. This corresponds to $l=0$ and $p_{z}=0$ for which the solution can be written as [27]

$$
\phi_{c}=v_{0} e^{-q B\left(x^{2}+y^{2}\right) / 4},
$$

where $v_{0}$ can be determined from the normalization condition. The corresponding ground state energy, or effective mass squared, is given by

$$
\mathcal{E}_{0}^{2}(0)=m^{2}+q B-\mu^{2} .
$$

As anticipated, the solution in Eq. (7) is not spatially uniform. In order to define an appropriate order parameter, we first normalize the solution over a given spatial volume $V$. This procedure involves finding the average over $V$ of $\phi_{c}^{2}$ defined as

$$
\left\langle\phi_{c}^{2}\right\rangle=\frac{1}{V} \int d^{3} x \phi_{c}^{2} .
$$

From Eqs. (7) and (9) one finds

$$
\left\langle\phi_{c}^{2}\right\rangle=v_{0}^{2}\left(\frac{1-e^{-\Phi / 2 \Phi_{0}}}{\Phi / 2 \Phi_{0}}\right),
$$

where $\Phi \equiv B A$ is the magnetic flux passing through the transverse area $A$ and $\Phi_{0} \equiv \pi / q$ is the quantum magnetic flux. Notice that for the analysis, neither $A$ nor $B$ can be taken as changing independently but instead that $\Phi$ should be considered as the relevant variable. Also, notice that the term between the parentheses in Eq. (10) goes to 1 as $\Phi \rightarrow 0$, as expected. The requirement to obtain the effective mass squared independent of the magnetic flux, leads us to consider $\bar{\phi}_{c} \equiv \sqrt{\left\langle\phi_{c}^{2}\right\rangle}$ as the order parameter to describe the condensation transition. This is determined as follows. In terms of $\bar{\phi}_{c}$ the ground state solution reads as

$$
\phi_{c}=\bar{\phi}_{c}\left(\frac{\Phi / 2 \Phi_{0}}{1-e^{-\Phi / 2 \Phi_{0}}}\right)^{1 / 2} e^{-q B\left(x^{2}+y^{2}\right) / 4} .
$$

We now look for the value of $\bar{\phi}_{c}$ that minimizes the classical action, this time accounting for the effects of the selfinteraction $(\lambda \neq 0)$. Substituting Eq. (11) into Eq. (4) we get

$$
\Gamma_{c}=\beta V\left[\frac{1}{2}\left(q B+m^{2}-\mu^{2}\right)\left\langle\phi_{c}^{2}\right\rangle+\frac{\lambda}{16}\left\langle\phi_{c}^{4}\right\rangle\right],
$$

where in general one defines

$$
\begin{aligned}
\left\langle\phi_{c}^{n}\right\rangle & \equiv \frac{1}{V} \int d^{3} x \phi_{c}^{n} \\
& =v_{0}^{n}\left(\frac{1-e^{-n \Phi / 4 \Phi_{0}}}{n \Phi / 4 \Phi_{0}}\right) .
\end{aligned}
$$

Therefore, the nontrivial minimum of Eq. (12) is found for a value of the order parameter $\bar{\phi}_{c}$ given explicitly by

$$
\begin{aligned}
\bar{\phi}_{c 0} & =4 \sqrt{\frac{\mu^{2}-m^{2}-q B}{\lambda}}\left(\frac{\Phi_{0}}{\Phi}\right)^{1 / 2} \\
& \times\left(\frac{1-e^{-\Phi / 2 \Phi_{0}}}{1+e^{-\Phi / 2 \Phi_{0}}}\right)^{1 / 2} \theta\left(\mu^{2}-m^{2}-q B\right) .
\end{aligned}
$$

Notice that for a given value of $B$ (and of $\Phi$ ), since the thermal occupation of the ground state grows when $\mu^{2} \rightarrow m^{2}+q B$, then Eq. (14) means that a macroscopic fraction of the charged particles will occupy the condensed state as $\bar{\phi}_{c 0} \rightarrow 0^{+}$. One can expect that the same is true when considering a higher order $n$ in the perturbative expansion of the effective action; that is, that the superfluid transition is signaled by the condition that $\bar{\phi}_{c n} \rightarrow 0^{+}$. We now proceed to find how this condition is realized at order $n=1$. 


\section{ONE-LOOP EFFECTIVE POTENTIAL}

The corrections to the value that minimizes the action are obtained from the grand potential. For the theory at hand, this is given by

$$
\Omega\left(\bar{\phi}_{c}\right)=-\frac{1}{\beta V} \ln \int D \tilde{\phi}^{*} D \tilde{\phi} e^{-S\left[\phi^{*}, \phi\right]},
$$

where $S\left[\phi^{*}, \phi\right]$ is the action defined in Eq. (2) and $\phi$ and $\tilde{\phi}$ are related as in Eq. (3). At one-loop order, the grand potential has the explicit expression

$$
\Omega\left(\bar{\phi}_{c}\right)=\left(m_{B}^{2}-\mu^{2}\right)\left\langle\phi_{c}^{2}\right\rangle+\frac{\lambda}{4}\left\langle\phi_{c}^{4}\right\rangle+\frac{1}{2 \beta V} \ln \operatorname{det} \mathbb{D}^{-1},
$$

where the inverse propagator matrix operator is defined as

$$
\mathbb{D}^{-1}=\left(\begin{array}{cc}
-\mathcal{D}_{-}^{2}+m^{2}+\frac{\lambda}{2} \phi_{c}^{2} & \frac{\lambda}{4} \phi_{c}^{2} \\
\frac{\lambda}{4} \phi_{c}^{2} & -\mathcal{D}_{+}^{2}+m^{2}+\frac{\lambda}{2} \phi_{c}^{2}
\end{array}\right),
$$

with

$$
\mathcal{D}_{ \pm}^{2}=\left(\partial_{\tau} \pm \mu\right)^{2}+(\boldsymbol{\nabla} \pm i q \boldsymbol{A})^{2} .
$$

Hereafter we use the notation

$$
m_{B} \equiv \sqrt{m^{2}+q B}
$$

for the effective mass in the lowest Landau level.

The fact that the operators $\mathcal{D}_{ \pm}^{2}$ depend on the coordinates makes it difficult to find the functional determinant. Nevertheless, since our interest is to explore the condensation phenomenon near the phase transition, we can resort to expand the grand potential in powers of the order parameter near the value $\bar{\phi}_{c 1}$ that minimizes it:

$$
\Omega\left(\bar{\phi}_{c}\right) \approx \Omega\left(\bar{\phi}_{c 1}\right)+\left.\frac{1}{2}\left(\bar{\phi}_{c}-\bar{\phi}_{c 1}\right)^{2}\left(\frac{\partial^{2} \Omega}{\partial \bar{\phi}_{c}^{2}}\right)\right|_{\bar{\phi}_{c}=\bar{\phi}_{c 1}}
$$

The factor

$$
\left.\mathcal{M}^{2}\left(\bar{\phi}_{c 1}\right) \equiv\left(\frac{\partial^{2} \Omega}{\partial \bar{\phi}_{c}^{2}}\right)\right|_{\bar{\phi}_{c}=\bar{\phi}_{c 1}}
$$

can be regarded as an effective mass squared for the quantum field $\tilde{\phi}$. Following the discussion at the end of Sec. II let us explore the behavior of $\Omega\left(\bar{\phi}_{c 1}\right)$ and $\mathcal{M}^{2}\left(\bar{\phi}_{c 1}\right)$ near the superfluid phase transition where we expect that $\bar{\phi}_{c 1} \rightarrow 0^{+}$. Notice also that $\mathcal{M}^{2}$ represents the curvature of the grand potential in the direction of $\bar{\phi}_{c}$. For a given value of $B$ (and of $\Phi$ ) the system is in the normal phase when the curvature is positive. As the occupation number of the ground state increases, the curvature should tend to change sign. Therefore the transition to the superfluid phase is also signaled by the condition $\mathcal{M}^{2} \rightarrow 0^{+}$. The explicit one-loop expressions for $\Omega(0)$ and $\mathcal{M}^{2}(0)$ are

$$
\begin{aligned}
\Omega(0) & =T \sum_{n=-\infty}^{\infty} \int \frac{d^{3} p}{(2 \pi)^{3}} \ln D^{-1} \\
\mathcal{M}^{2}(0) & =m_{B}^{2}-\mu^{2}+\lambda T \sum_{n=-\infty}^{\infty} \int \frac{d^{3} p}{(2 \pi)^{3}} D,
\end{aligned}
$$

where $D$ is the propagator for a charged scalar in the presence of a constant magnetic field. We use the expression for $D$ obtained in the Schwinger proper time method, given by

$$
D=\int_{0}^{\infty} \frac{d s}{\cosh (q B s)} e^{-s\left[\left(\omega_{n}-i \mu\right)^{2}+p_{z}^{2}+m^{2}+p_{\perp}^{2} \frac{\tanh (q B s)}{q B s}\right]},
$$

where $p_{\perp}^{2}$ represents the square of the components of $\boldsymbol{p}$ transverse to the direction of the magnetic field and $\omega_{n}$ is a boson Matsubara frequency. Also, in writing Eq. (23) we have ignored a phase factor which does not contribute when considering closed loop expressions. Note that the one-loop correction in $\mathcal{M}^{2}(0)$ is in fact the self-energy. This is not the case when one considers higher loop corrections. Carrying out the integrations over the transverse components we get

$$
\begin{aligned}
\Omega(0) & =\frac{q B T}{2 \pi} \sum_{l, n} \int \frac{d p_{z}}{2 \pi} \ln \left(P^{2}+m^{2}\right) \\
\mathcal{M}^{2}(0) & =m_{B}^{2}-\mu^{2}+\lambda \frac{q B T}{2 \pi} \sum_{l, n} \int \frac{d p_{z}}{2 \pi} \frac{1}{P^{2}+m^{2}}
\end{aligned}
$$

where

$$
P^{2}=\left(\omega_{n}-i \mu\right)^{2}+p_{z}^{2}+q B(2 l+1)
$$

with $l \geq 0$ being the index labeling the Landau levels.

Let us now separate the $T=0$ (purely magnetic field) contribution from the thermal dependence by writing

$$
\begin{aligned}
\Omega(0) & =\Omega_{B}+\Omega_{T, B} \\
\mathcal{M}^{2}(0) & =m_{B}^{2}-\mu^{2}+\Pi_{B}+\Pi_{T, B}
\end{aligned}
$$

where the subscripts $T$ and $B$ denote the temperature and magnetic field-dependent contributions.

Let us first compute the purely magnetic field contributions. As shown in the Appendix, these contributions are easily obtained using dimensional regularization. They can be expressed in terms of the Hurwitz zeta function $\zeta(s, u)$ and are given explicitly by

$$
\begin{aligned}
\Omega_{B} & =\frac{m^{4}}{(4 \pi)^{2}}\left[\frac{1}{2} \ln \left(\frac{2 q B}{m^{2}}\right)\right. \\
& \left.+\left(\frac{2 q B}{m^{2}}\right)^{2} \zeta^{\prime}\left(-1, \frac{1}{2}+\frac{m^{2}}{2 q B}\right)\right] \\
\Pi_{B} & =\frac{\lambda m^{2}}{(4 \pi)^{2}}\left[1+\ln \left(\frac{2 q B}{m^{2}}\right)\right. \\
& \left.+\left(\frac{2 q B}{m^{2}}\right) \ln \left\{\Gamma\left(\frac{1}{2}+\frac{m^{2}}{2 q B}\right) / \sqrt{2 \pi}\right\}\right],
\end{aligned}
$$

where $\zeta^{\prime}(s, u)=\partial \zeta(s, u) / \partial s$ and $\Gamma(u)$ is the gamma function. In writing Eq. (27) we have chosen the renormalization scale in the $\overline{\mathrm{MS}}$ scheme as $\Lambda_{\overline{\mathrm{MS}}}=m e^{-1 / 2}$. With this choice, one gets a vanishing contribution in the limit $B \rightarrow 0$. 
As is also shown in the Appendix, the thermal contribution to $\Omega(0)$ and $\mathcal{M}^{2}(0)$ can be expressed as

$$
\begin{aligned}
& \Omega_{T, B}=-\frac{q B}{4 \pi^{2}} \sum_{n=1}^{\infty} \cosh (\beta \mu n) \int_{0}^{\infty} \frac{d s}{s^{2}} \frac{e^{-s m_{B}^{2}-\beta^{2} n^{2} / 4 s}}{1-e^{-2 q B s}}, \\
& \Pi_{T, B}=\lambda \frac{q B}{4 \pi^{2}} \sum_{n=1}^{\infty} \cosh (\beta \mu n) \int_{0}^{\infty} \frac{d s}{s} \frac{e^{-s m_{B}^{2}-\beta^{2} n^{2} / 4 s}}{1-e^{-2 q B s}} .
\end{aligned}
$$

Since condensation is a low temperature phenomenon, let us approximate Eq. (28) in the limit where $T \ll m_{B}$. In this case, the integrals can be computed using the steepest descent method and the result can be expressed as

$$
\begin{aligned}
\Omega_{T, B} & \approx-2 \pi m_{B}^{4} \tau^{5 / 2}\left[\gamma L i_{3 / 2}(z)+\sum_{n=1}^{\infty} \frac{z^{n}}{n^{5 / 2}} \frac{n \gamma}{e^{n \gamma}-1}\right] \\
& +\{\mu \rightarrow-\mu\}, \\
\Pi_{T, B} & \approx \frac{\lambda}{2} m_{B}^{2} \tau^{3 / 2}\left[\gamma L i_{1 / 2}(z)+\sum_{n=1}^{\infty} \frac{z^{n}}{n^{3 / 2}} \frac{n \gamma}{e^{n \gamma}-1}\right] \\
& +\{\mu \rightarrow-\mu\},
\end{aligned}
$$

where the polylogarithm function is defined as

$$
L i_{s}(z) \equiv \sum_{n=1}^{\infty} \frac{z^{n}}{n^{s}}
$$

and the fugacity $z$, scaled temperature $\tau$ and scaled magnetic field $\gamma$ are defined as

$$
\begin{aligned}
z & \equiv e^{\left(\mu-m_{B}\right) / T}, \\
\tau & \equiv \frac{T}{2 \pi m_{B}}, \\
\gamma & \equiv \frac{q B}{m_{B} T}
\end{aligned}
$$

respectively. Notice that the sum over the index $n$ in Eq. (29) corresponds to a sum over Matsubara frequencies. Also, in writing Eq. (29) from Eq. (28), we have explicitly separated the contribution from the lowest Landau level - whose expression is given in terms of the polylogarithm function - from the contribution of the rest of the energy levels. This separation proves useful since the contribution from the lowest Landau level, unlike that from the rest of the levels, is strongly infrared divergent near the phase transition and must be treated separately [21].

Recall that the charge density is defined as

$$
\rho=-\frac{\partial \Omega\left(\bar{\phi}_{c}\right)}{\partial \mu}
$$

Near the superfluid transition where $\mathcal{M}^{2}(0) \rightarrow 0$ one can compute the charge density by considering only the term $\Omega(0)$ in the grand potential, therefore, at one-loop order, the thermal part of the charge density is obtained from the first line of Eq. (29) as

$$
\begin{aligned}
\rho & \approx m_{B}^{3} \tau^{3 / 2}\left[\gamma L i_{1 / 2}(z)+\sum_{n=1}^{\infty} \frac{z^{n}}{n^{3 / 2}} \frac{n \gamma}{e^{n \gamma}-1}\right] \\
& -\{\mu \rightarrow-\mu\} .
\end{aligned}
$$

Since the phase transition happens when $\mu \sim m_{B}$ the terms with $\{\mu \rightarrow-\mu\}$ in Eqs. (29) and (35) are negligible, given that they become proportional to powers of the factor $\exp \left(-\left(m_{B}+\mu\right) / T\right) \sim \exp \left(-2 m_{B} / T\right) \ll 1$, and thus hereafter we ignore them. Notice that with this approximation, the second equation of Eq. (29) and Eq. (35) imply that $\Pi_{T, B}$ and $\rho$ are proportional. We have now set up the stage to discuss the condensation phenomenon in terms of a finite charge density in the ground state.

\section{BOSE-EINSTEIN CONDENSATION REVISITED}

The existence of a critical temperature and a critical chemical potential indicates that the system of charged bosons reaches a kind of saturation where the occupation of the ground state becomes important. This saturation leads to the superfluidity phenomenon. From the computational point of view, the condensation conditions are searched for from the values of the parameters that minimize the vacuum energy. Given that the description of the onset of condensation may be obscured by the existence of infrared divergent quantities and in order to gain insight, let us first revisit how these conditions are found at tree and one-loop level.

At tree level, the condensation condition is given by $\mu=m_{B}$. This condition implies the vanishing of the mass term $\mathcal{M}^{2}(0)$ in the grand potential, as can be seen from Eq. (26). If we now consider the one-loop correction, from the second equation of Eq. (29) and from the limiting behavior of $L i_{1 / 2}(z)$ for $\mu \rightarrow m_{B}(z \sim 1)$ and $\mu \ll m_{B}(z \ll 1)$

$$
L i_{1 / 2}(z) \approx\left\{\begin{array}{cl}
\sqrt{\frac{\pi}{1-z}}+\zeta\left(\frac{1}{2}\right) & \text { for } z \lesssim 1 \\
z & \text { for } z \ll 1
\end{array}\right.
$$

we see that $\mathcal{M}^{2}(0)$ diverges when $\mu \rightarrow m_{B}$. This behavior has been interpreted as the impossibility of the existence of a superfluid state in the presence of an external magnetic field [17]. However, this usual prescription for the onset of condensation, is not adequate in the presence of an external magnetic field.

Consider the situation in the absence of a magnetic field. Intuitively, once the superfluid phase is established, it is difficult to imagine that this can be instantaneously destroyed by the turning on of an arbitrary small external magnetic field. One would expect that in case the magnetic field destroys the superfluid state, when the field is small, the condensed state should be restored for a different temperature. Similar considerations were made 
for the case of a noninteracting gas in Ref. 21] albeit for a high external magnetic field, in the limit $T \rightarrow 0$. In such case it was shown that the charge density in the normal phase vanishes, i.e., all the charges populate the superfluid phase. However, since the physical conditions require to have a finite charge density, the chemical potential - which depends on temperature as well as on this charge density - does not reach the value that corresponds to the ground state energy and therefore no infrared divergence occurred. It is important to emphasize that Ref. 21] suggests that under such conditions, there is no definite critical temperature associated with the superfluid transition.

To continue gaining insight, let us keep on analyzing the case with zero external magnetic field. From Eq. (35), the charge density for $\gamma \rightarrow 0$ becomes

$$
\rho \approx\left(\frac{m T}{2 \pi}\right)^{3 / 2} L i_{3 / 2}\left(e^{(\mu-m) / T}\right)
$$

The function $L i_{3 / 2}(z)$ is well defined for $z \leq 1$, although its derivative diverges at $z=1$. Notice that there is no analytical continuation for $L i_{3 / 2}(z)$ which gives a real result for $z>1$. We have explicitly

$$
L i_{3 / 2}(z) \approx\left\{\begin{array}{cl}
\zeta\left(\frac{3}{2}\right)+\sqrt{4 \pi(1-z)} & \text { for } z \lesssim 1 \\
z & \text { for } z \ll 1
\end{array}\right.
$$

Thus, the maximum value allowed for the chemical potential is $\mu_{0}=m(z=1)$. This result is interpreted as the saturation of the boson system that gives rise to condensation at this critical value of the chemical potential. Since the charge density is a conserved quantity, there must be a critical temperature $T_{0}$ for which condensation takes place. In other words $\mu_{0}=\mu\left(T_{0}, \rho\right)$. The condition to obtain the critical temperature comes from Eq. (37) by setting $\mu_{0}=m$, resulting in

$$
T_{0}=\frac{2 \pi}{m}\left(\frac{\rho}{\zeta\left(\frac{3}{2}\right)}\right)^{2 / 3} .
$$

This is the well-known result for the critical temperature of a noninteracting boson gas. For temperatures lower than the critical temperature, $T<T_{0}$, the gas can be separated into two phases: the normal $(N)$ phase and the superfluid $(S)$ phase. The charge density splits into these two states $\rho=\rho_{N}+\rho_{S}$, where the charge density in the normal phase $\rho_{N}$ is defined as the charge density $\rho$, evaluated at the critical chemical potential $\mu_{0}$. In the absence of the magnetic field this is given by

$$
\rho_{N}=\left(\frac{m T}{2 \pi}\right)^{3 / 2} \zeta(3 / 2)
$$

The superfluid charge density corresponds to the difference between the total charge density and the charge density in the normal phase, $\rho_{S}=\rho-\rho_{N}$, for a fixed total charge density $\rho$.

When interactions are accounted for, the situation changes. The condition for the phase transition is once again looked for from the vanishing of the effective mass squared $\mathcal{M}^{2}(0)$. From Eq. (26) and the second equation of Eq. (29), in the limit $B \rightarrow 0$, the effective mass squared is given by

$$
\begin{aligned}
\mathcal{M}^{2}(0) & =m^{2}-\mu^{2} \\
& +\frac{\lambda m^{2}}{2}\left(\frac{T}{2 \pi m}\right)^{3 / 2} L i_{3 / 2}\left(e^{(\mu-m) / T}\right) \\
& \stackrel{\mu \rightarrow m}{\longrightarrow} \frac{\lambda m^{2}}{2}\left(\frac{T}{2 \pi m}\right)^{3 / 2} \zeta(3 / 2),
\end{aligned}
$$

which shows that $\mathcal{M}^{2}(0)$ cannot vanish for $\mu \leq m$. Moreover, since the function $L i_{3 / 2}(z)$ cannot be analytically continued to real values for $z>1$, there is no physical solution that sets $\mathcal{M}^{2}(0)=0$ even if we were to consider $\mu>m$.

The above results show the need of an extra ingredient already for $B=0$. In this case, the solution is well known: since the physical conditions require the effective mass squared to vanish, plasma screening effects need to be accounted for by means of resummation. Examples of the importance to include resummation effects have been recently discussed for systems subject to the influence of an external magnetic field. For instance in Ref. 28] the temperature dependent effective potential for a scalar theory, similar to the case here discussed, was considered. A resummation of ring diagrams turns out to be extremely important to understand the appearance of a first order phase transition. The scenario has also been considered in other theories such as the linear sigma model [29] and the standard model, during the electroweak phase transition [30]. In all these cases, a second order phase transition turns into a first order one.

In the present context inclusion of resummation effects means that the self-energy should be computed selfconsistently as

$$
\Pi \rightarrow \bar{\Pi}=\lambda T \sum_{n=-\infty}^{\infty} \int \frac{d^{3} p}{(2 \pi)^{3}} D\left(m^{2} \rightarrow m^{2}+\bar{\Pi}\right) .
$$

To simplify the calculation, and in case the coupling $\lambda$ is not too large, it is customary to substitute inside the argument of the propagator in Eq. (42), $\bar{\Pi} \rightarrow \Pi_{1}$. For the low temperature expansion for $B=0$ and when $\mu=m$, we see from the second equation of Eq. (29) and Eq. (38) that in this case

$$
\Pi_{1}=\frac{\lambda m^{2}}{2}\left(\frac{T}{2 \pi m}\right)^{3 / 2} \zeta(3 / 2) .
$$

When the coupling $\lambda$ is not small, a full self-consistent treatment is needed.

In the following section we explain in detail the resummation procedure for a finite external magnetic field. From there, the case treated in this section is obtained as the limit with $B \rightarrow 0$. 


\section{RESUMMATION AT FINITE $B$}

As was mentioned in the previous section, in order to consistently compute the critical temperature when interactions are accounted for, it is necessary to consider resummation. Inclusion of these effects allows us to find the chemical potential, beyond its tree level value $\mu=m$, by consistently accounting for plasma screening. The need for resummation is even more dramatic for $B \neq 0$, since when ignoring screening and $\mu \rightarrow m_{B}$ the charge density and the thermal contribution to the self-energy both diverge in the infrared. In Ref. 31] the thermal effective potential in the $\lambda \phi^{4}$ theory was computed by a resummation of the ring diagrams. Effectively, the resummation was done through a solution of the renormalization group equation. Resummation results in a shift of $m^{2}$ in the one-loop correction of the grand potential

$$
\begin{aligned}
\Omega(0) \rightarrow \Omega_{r}(0)= & \Omega_{B}+\left.\Omega_{T, B}\right|_{m^{2} \rightarrow m^{2}+\bar{\Pi}} \\
\mathcal{M}^{2}(0) \rightarrow \mathcal{M}_{r}^{2}(0)= & m_{B}^{2}-\mu^{2} \\
& +\Pi_{B}+\left.\Pi_{T, B}\right|_{m^{2} \rightarrow m^{2}+\bar{\Pi}}
\end{aligned}
$$

where the subscript $r$ is to emphasize that resummation effects are included. As a result, the charge density becomes

$$
\rho=-\frac{\partial \Omega_{r}(0)}{\partial \mu}
$$

The resummed self-energy $\bar{\Pi}$ is obtained self-consistently

$$
\begin{aligned}
\bar{\Pi} & =\left.\lambda \frac{q B T}{2 \pi} \sum_{l, n} \int \frac{d p_{z}}{2 \pi} \frac{1}{P^{2}+m^{2}+\bar{\Pi}}\right|_{\mu=m_{B}} \\
& -\{T \rightarrow 0\},
\end{aligned}
$$

where $P^{2}$ is defined in Eq. (25). We consider only the thermal contribution, as indicated by subtracting the $\{T \rightarrow 0\}$ term. Also since we are interested in computing this self-energy near the phase transition, we consider its value for $\mu=m_{B}$. The self-consistent equation is reduced to finding the solution to the expression

$$
\bar{\Pi}=\left.\Pi_{T, B}\right|_{m^{2} \rightarrow m^{2}+\bar{\Pi}, \mu=m_{B}} .
$$

For the rest of this section, we concentrate on finding an explicit expression for $\bar{\Pi}$ in some limits.

In order to simplify the self-consistent equation, recall that since we want to include the effect of thermal fluctuations coming from the resummation of the so-called ring diagrams for small temperatures, we use the low temperature approximation for $\Pi_{T, B}$ given in Eq. (29). We can consider $\bar{\Pi} \ll m^{2}$ up to the leading order in the righthand side of Eq. (47). At leading order, we can set $\bar{\Pi}=0$ in all the terms, except in the argument

$$
z=\left.e^{\left(\mu-\sqrt{m^{2}+q B}\right) / T}\right|_{m^{2} \rightarrow m^{2}+\bar{\Pi}, \mu \rightarrow m_{B}}
$$

of the polylogarithm function $L_{1 / 2}(z)$, since this diverges when $\mu=m_{B}$ in the limit $\bar{\Pi} \rightarrow 0$. Therefore we set

$$
\begin{aligned}
z & \rightarrow e^{\left(m_{B}-\sqrt{m^{2}+\bar{\Pi}+q B}\right) / T} \\
& \approx e^{-\bar{\Pi} / 2 m_{B} T} .
\end{aligned}
$$

We explore the situation where $\bar{\Pi} \ll m_{B} T$, which is a good enough condition to control the infrared divergences. Then we can expand the fugacity as

$$
z \approx 1-\Pi / 2 m_{B} T \text {. }
$$

Using the expression for $L i_{1 / 2}$ in Eq. (36) we obtain a simplified expression for the self-consistent equation

$$
\bar{\Pi} \approx \frac{\lambda}{2} m_{B}^{2} \tau^{3 / 2}\left[\gamma \sqrt{\frac{2 \pi m_{B} T}{\bar{\Pi}}}+\sum_{n=1}^{\infty} \frac{1}{n^{3 / 2}} \frac{n \gamma}{e^{n \gamma}-1}\right] .
$$

This is a third order equation in the variable $\sqrt{\bar{\Pi}}$. It can be solved explicitly, though the solution is given in terms of complicated expressions. It is therefore more instructive to find the solution for different values of $T$ and $B$, in particular, the next-to-leading order correction in $\lambda$ for the case $q B \ll m_{B} T$ here treated. For this purpose, we expand Eq. (51) for $\gamma \rightarrow 0$. Also, since we are close to $B=0$ we can write $\Pi_{1}$, given in Eq. (43), instead of $\bar{\Pi}$ in the right-hand side of Eq. (51), resulting in

$$
\bar{\Pi} \approx \frac{\lambda^{1 / 2} q B}{\sqrt{2 \zeta(3 / 2)}}\left(\frac{T}{2 \pi m}\right)^{1 / 4}+\frac{\lambda m^{2}}{2}\left(\frac{T}{2 \pi m}\right)^{3 / 2} \zeta(3 / 2) .
$$

It can be shown that the first term on the right-hand side of Eq. (52) is the leading correction coming from the lowest Landau level. Notice that this term also makes explicit the breaking of the perturbative regime as it is proportional to $\lambda^{1 / 2}$.

We emphasize that resummation is needed only for the case when considering small values of the external magnetic field, compared with temperature. In fact, notice that the nonthermal contribution to the self-energy $\Pi_{B}$ in Eq. (27) is negative. Its limiting values can be approximated as

$$
\Pi_{B} \approx \begin{cases}-\frac{\lambda}{6}\left(\frac{q B}{4 \pi m}\right)^{2} & \text { for } q B<m^{2} \\ -\frac{\lambda q B}{(4 \pi)^{2}} \ln 2 & \text { for } q B \gg m^{2} .\end{cases}
$$

Therefore, in this case there is always a sufficiently small temperature such that $\Pi_{B}+\Pi_{T, B}<0$ for $\mu \lesssim m_{B}$ and the effective mass squared $\mathcal{M}(0)^{2}$ in Eq. (26) can thus vanish.

\section{CRITICAL TEMPERATURE}

Having solved the infrared divergence problem by the introduction of the resummation procedure in the selfenergy, we now proceed to find the critical temperature for the superfluid phase transition and explore how 
this critical temperature is modified as a function of the strength of the magnetic field, compared to the zero field case. As we pointed out in Sec. IV, the charge density is a conserved quantity and therefore it cannot diverge. From the resummed version of Eq. (35), that is, with the mass shifted as $m^{2} \rightarrow m^{2}+\bar{\Pi}$, we can express the chemical potential in terms of the charge density and the other parameters, namely, $\mu=\mu(\rho, T, B)$. For a fixed charge density and a given value of the magnetic field, the critical temperature $T_{c}(\rho, B)$ can be found from the condition that sets the grand potential up to its minimal value. Recall that for $B=0$ it is well known that the phase transition is of the second order. Even more, in the case of zero chemical potential, the transition is always of second order 25]. Here we will assume that, for $B \neq 0$ the phase transition continues being of the second order, which is the expected behavior if we slowly turn on the magnetic field.

$$
\begin{aligned}
\left.\mathcal{M}_{r}^{2}(0)\right|_{T=T_{c}, \mu=\mu_{c}} & =0 \\
-\left.\frac{\partial \Omega_{r}(0)}{\partial \mu}\right|_{T=T_{c}, \mu=\mu_{c}} & =\rho,
\end{aligned}
$$

where $\mu_{c}(\rho, B) \equiv \mu\left(T_{c}, \rho, B\right)$. We proceed to numerically solve Eq. (54).

In the low temperature approximation that we are considering, the thermal contribution to the self-energy is proportional to the charge density, as can be seen from Eqs. (29) and (35), upon neglecting the terms with $\{\mu \rightarrow-\mu\}$. Therefore we can write

$$
\Pi_{T, B}=\frac{\lambda \rho}{2 m_{B}} .
$$

The same is true after including resummation effects. Since $\rho$ is constant, we can use the above result to immediately solve for the critical chemical potential from the first line of Eq. (54), resulting in

$$
\mu_{c} \approx\left[m_{B}^{2}+\Pi_{B}+\frac{\lambda \rho}{2 \sqrt{m_{B}^{2}+\bar{\Pi}}}\right]^{1 / 2} .
$$

Notice that now, finding the critical temperature becomes equivalent to solving Eq. (35) with the replacement $m^{2} \rightarrow m^{2}+\bar{\Pi}$, where for $T$ and $\mu$ use is made of their critical values $T=T_{c}, \mu=\mu_{c}$, with $\mu_{c}$ given explicitly in Eq. (56). Let us now find $T_{c}$ numerically. For this purpose, we consider a charged pion condensate in the core of compact stars. The mass is given by $m=m_{\pi} \approx 140$ $\mathrm{MeV}$. The typical surface temperature for a cold neutron star is $T \sim 100 \mathrm{eV}$. Since the critical temperature $T_{c}$ for small $B$ is expected to be close to the value $T_{c 0}$ at zero magnetic field, we can use Eq. (39) to obtain the charge density that is needed for the onset of condensation at these temperatures.

Figures 1 and 2 show the critical temperature $T_{c}$ scaled to the critical temperature at zero magnetic field $T_{c 0}$ as a
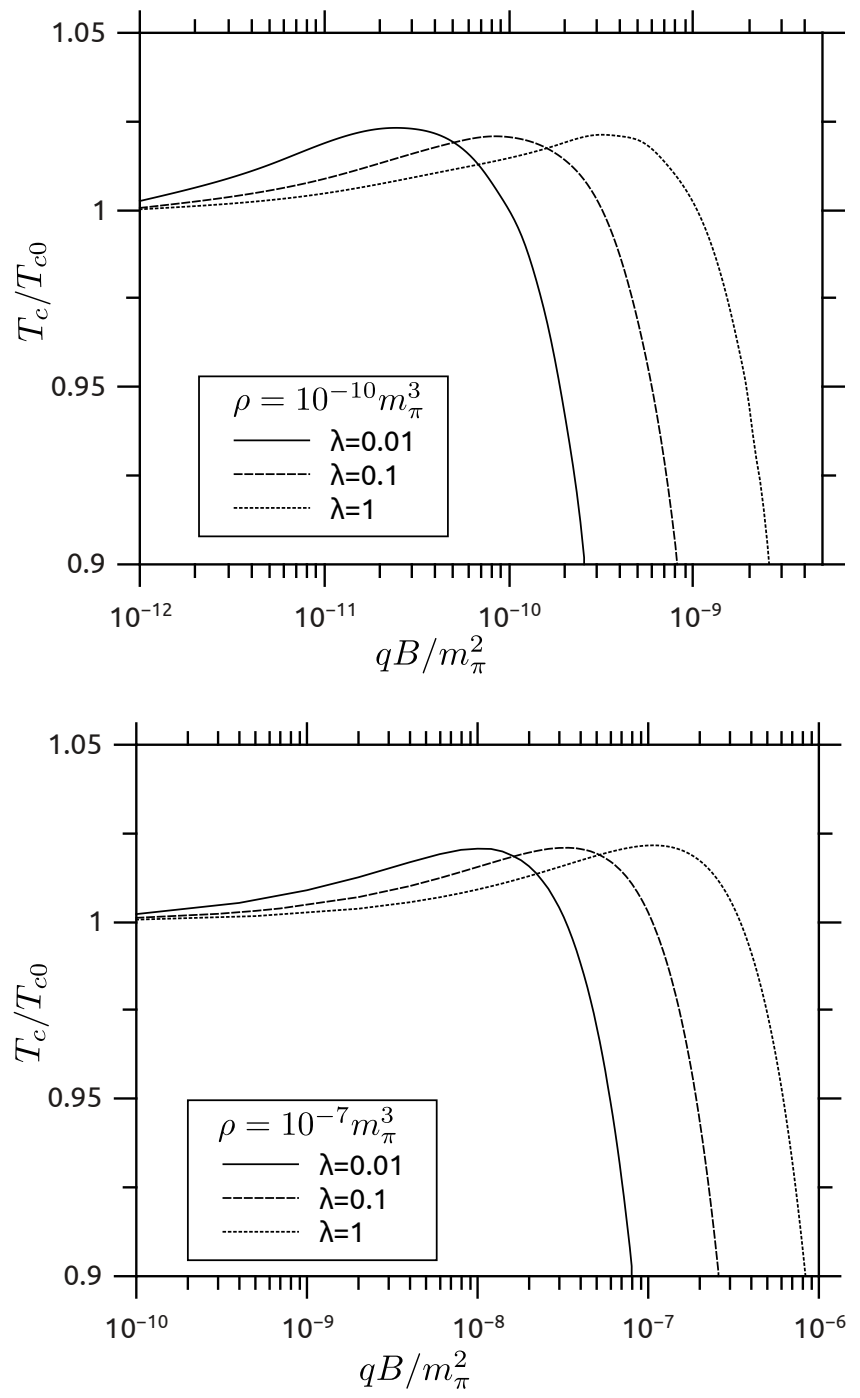

FIG. 1. Critical temperature $T_{c}$ scaled to the critical temperature $T_{c 0}$ as a function of the magnetic field strength scaled to $m_{\pi}^{2}$. The calculation is valid for the case when $q B \ll m_{B} T$. The upper panel corresponds to a density $\rho=10^{-10} m_{\pi}^{3}$ whereas the lower panel to a density $\rho=10^{-7} \mathrm{~m}_{\pi}^{3}$.

function of the magnetic field strength scaled to $m_{\pi}^{2}$. In both cases we consider two different values of the charge density, $\rho=10^{-10} m_{\pi}^{3}$ and $\rho=10^{-7} m_{\pi}^{3}$, which correspond roughly to temperatures $T_{0} \sim 100 \mathrm{eV}$ and $T_{0} \sim 10$ $\mathrm{keV}$, respectively.

Figure 1 corresponds to the case $q B \ll m_{B} T$. In practice we consider that the parameter $\gamma$ defined in Eq. (33) is such that $\gamma<10^{-3}$. Notice the smooth rise of the critical temperature as the magnetic field increases. This signals that when the magnetic field is slowly turned on it catalyzes the formation of the condensate; that is, the critical temperature is larger than for the $B=0$ case. Nevertheless this behavior disappears for higher values of the magnetic field and the formation of the condensate appears at lower temperatures. We also mention that catalysis in this case can occur for a larger range of mag- 

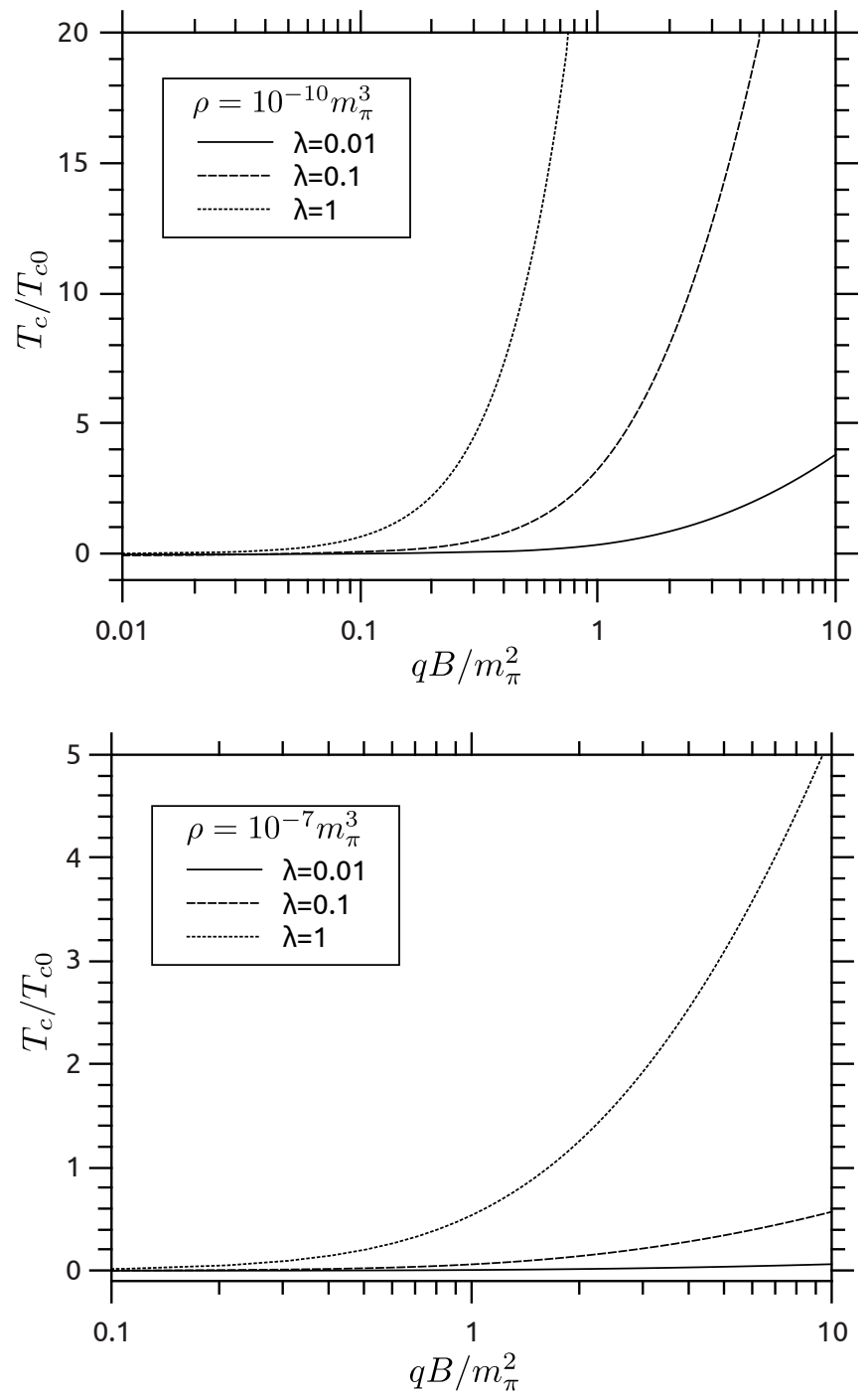

FIG. 2. Critical temperature $T_{c}$ scaled to the critical temperature $T_{c 0}$ as a function of the magnetic field strength scaled to $m_{\pi}^{2}$. The calculation is valid for the case when $q B \gg m_{B} T$. The upper panel corresponds to a density $\rho=10^{-10} m_{\pi}^{3}$ whereas the lower panel to a density $\rho=10^{-7} m_{\pi}^{3}$.

netic field strengths when the charge density is larger, as well as for larger values of the coupling constant $\lambda$. Figure 2 corresponds to the case $q B \gg m_{B} T$. We explicitly consider $\gamma>10^{3}$. For these larger values of $B$ the critical temperature grows. This means that at some intermediate values of $q B$ between the large and small regions here considered, the magnetic field catalyzes again the formation of the condensate. The catalysis is enhanced as the coupling constant $\lambda$ increases, and contrary to the low magnetic field case, it is also enhanced for lower densities.

\section{SUMMARY AND CONCLUSIONS}

In this work we have studied the condensation phenomenon for a system of charged bosons in the presence of an external magnetic field. Contrary to what is commonly believed, we have shown that condensation happens at a definite critical temperature. The missing ingredient, overlooked in previous analysis and accounted for in this work, is the treatment of the plasma screening effects by means of resummation. We have explicitly computed the critical temperature, for typical densities found in compact astrophysical objects, for small and large values of the magnetic fields. We have shown that the magnetic field catalyzes the onset of the condensation at very small and large values of the magnetic field, agreeing in these regions with the case of zero chemical potential 25]. For intermediate values of the magnetic field, the critical temperature for condensation turns out to be lower than in the $B=0$ case.

Recall that although the term magnetic catalysis usually refers to an enhancement of dynamical symmetry breaking by an external magnetic field, the phenomenon seems to be universal, appearing in different physical scenarios [32] such as the one treated in this work, namely, a condensate of scalar particles, as opposed to the more usually studied case of a fermion condensate.

We should also mention that the problem has been studied by lattice methods as well. The first lattice simulation for deconfinement and chiral symmetry restoration for two-flavor QCD, in the presence of a magnetic background, was done in Refs. 33, 34]. The result was that the transition temperature significantly decreased with increasing magnetic field. A similar conclusion was presented in 35]. In that work it was found that in the chirally broken phase, the chiral condensate increased monotonically with a growing magnetic field strength. In fact, in the chiral limit this behavior started linearly. In the same limit and in the chirally restored phase, the condensate vanished independent of the strength of the magnetic field. On the other hand, in Refs. [37, 38] the effect of an external magnetic field on the finite temperature transition of QCD was considered. Thermodynamic observables including the chiral condensate and the susceptibility were measured. The result was that the transition temperature significantly decreased with increasing magnetic field. Such discrepancies can be originated by the fact that in Refs. 33 35 the pion had a large mass. In the case of Refs. 37, 38 light pions as well as an improved lattice were used. These seemingly contrasting results call for a closer look at the phenomenon, in particular for the case of intermediate values of the magnetic field strength. This is work that we are currently pursuing and will be reported elsewhere. 


\section{ACKNOWLEDGMENTS}

A.A. thanks the faculty and staff of the physics department at PUC for their kind hospitality during a work visit in January 2012. Support for this work has been received in part from DGAPA-UNAM under Grant No. PAPIIT-IN103811 and CONACyT-México under Grant No. 128534. M.L., J.C.R and C.V. acknowledge support from FONDECYT under Grant No. 1095217. M.L and J.C.R. acknowledge support from FONDECYT under Grant No. 1120770. M.L. acknowledges support from Proyecto Anillos ACT 119 (Chile).

\section{Appendix}

The grand potential for a scalar field in the presence of a uniform magnetic field evaluated at $\phi_{c}=0$, is given by

$$
\begin{aligned}
\Omega(0) & =\frac{1}{2} \operatorname{tr} \ln \left(\mathcal{D}_{+}+m^{2}\right)+\frac{1}{2} \operatorname{tr} \ln \left(\mathcal{D}_{-}+m^{2}\right), \\
& =\int d m^{2} T \sum_{n=-\infty}^{\infty} \int \frac{d^{d} p}{(2 \pi)^{d}} D\left(\boldsymbol{k}, \omega_{n}\right),
\end{aligned}
$$

where the operators $\mathcal{D}_{ \pm}$are defined in Eq. (18) and the propagator $D$ expressed in the Schwinger proper time formalism is defined in Eq. (23). The validity of the second line in Eq. (A.1) is more easily seen from the representation of the grand potential in terms of Landau levels, Eq. (24). Thus, to obtain the grand potential, we need to compute the term proportional to the oneloop self-energy and then integrate over $m^{2}$, neglecting unphysical ultraviolet divergent constants.

The sum over Matsubara frequencies $\omega_{n}=2 \pi n T$ gives rise to the Jacobi theta function

$$
\theta_{3}(z, x)=\sum_{n=-\infty}^{\infty} e^{-\pi x n^{2}+2 \pi z n},
$$

which obeys the inversion property [36]

$$
\theta_{3}(z, x)=\frac{e^{\pi z^{2} / x}}{\sqrt{x}} \theta_{3}\left(\frac{z}{i x}, \frac{1}{x}\right) .
$$

Identifying $z=2 i T \mu s$ and $x=4 \pi T^{2} s$ allows us to rewrite the full propagator as

$$
\begin{aligned}
T \sum_{n=-\infty}^{\infty} D= & \frac{1}{\sqrt{\pi}} \int_{0}^{\infty} \frac{d s}{\sqrt{s}} \frac{e^{-s\left[m^{2}+p_{z}^{2}+p_{\perp}^{2} \frac{\tanh (e B s)}{e B s}\right]}}{\cosh (e B s)} \\
& \times\left[\frac{1}{2}+\sum_{n=1}^{\infty} e^{-\frac{\beta^{2} n^{2}}{4 s}} \cosh (\beta \mu n)\right] .
\end{aligned}
$$

The ultraviolet divergent term is the first term inside the square bracket. The one-loop self-energy, including the counterterm $\delta m^{2}$, is given by

$$
\begin{aligned}
\Pi & =\delta m^{2}+\lambda T \sum_{n=-\infty}^{\infty} \int \frac{d^{3} p}{(2 \pi)^{3}} D \\
& =\Pi_{B}+\Pi_{T, B},
\end{aligned}
$$

where we separate the thermal contribution $\Pi_{T, B}$ from the purely magnetic field contribution $\Pi_{B}$. This last contribution contains the ultraviolet divergence, which is canceled with the counterterm $\delta m^{2}$ using dimensional regularization [25]. Introducing the scale factor $\Lambda$, the purely magnetic contribution is given by

$$
\begin{aligned}
\Pi_{B}= & \delta m^{2}+\frac{\lambda}{\sqrt{4 \pi}} \int \frac{d^{d} p}{(2 \pi)^{d}} \Lambda^{3-d} \int_{0}^{\infty} \frac{d s}{\sqrt{s}} \\
& \times \frac{e^{-s\left[m^{2}+p_{z}^{2}+p_{\perp}^{2} \frac{\tanh (q B s s)}{q B s}\right]}}{\cosh (q B s)} .
\end{aligned}
$$

Integrating the $\boldsymbol{p}_{\perp}$ and $p_{z}$ momentum components and expanding in powers of $e^{-q B s}$, the purely magnetic contribution to the self-energy is expressed in terms of a sum over the Landau levels

$$
\begin{aligned}
\Pi_{B}= & \delta m^{2}+\frac{2 \lambda q B \Lambda^{d-3}}{(4 \pi)^{(d+1) / 2}} \sum_{l=0}^{\infty} \int_{0}^{\infty} d s \frac{e^{-s\left(m^{2}+q B(2 l+1)\right)}}{s^{(d-1) / 2}} \\
= & \delta m^{2}+\frac{2 \lambda q B}{(4 \pi)^{2}}\left(\frac{4 \pi \Lambda^{2}}{2 q B}\right) \\
& \times \zeta\left(\frac{3-d}{2}, \frac{1}{2}+\frac{m^{2}}{2 q B}\right) \Gamma\left(\frac{3-d}{2}\right),
\end{aligned}
$$

where $\zeta$ is the Hurwitz zeta function

$$
\zeta(s, u)=\sum_{l=0}^{\infty}(l+u)^{-s},
$$

Expanding in the number of dimensions $3-d$ we get

$$
\begin{aligned}
\Pi_{B}= & \delta m^{2}-\frac{\lambda m^{2}}{(4 \pi)^{2}}\left[\frac{2}{3-d}-\gamma+\ln \frac{4 \pi \Lambda^{2}}{2 q B}\right. \\
& \left.-\frac{2 e B}{m^{2}} \zeta^{\prime}\left(0, \frac{1}{2}+\frac{m^{2}}{2 e B}\right)\right],
\end{aligned}
$$

where $\zeta^{\prime}(s, u) \equiv \partial_{s} \zeta(s, u)$. The divergent term is removed using $\overline{\mathrm{MS}}$ scheme. In order to have a vanishing contribution to the self-energy in the absence of thermal and magnetic effect, one can choose for the scale factor the convenient value $\Lambda=m e^{-1 / 2}$. Using the relation $\zeta^{\prime}(0, u)=\ln (\Gamma(u) / \sqrt{2 \pi})$ we finally arrive at the expression for $\Pi_{B}$ in Eq. (27).

The expression for $\Omega_{B}$ in Eq. (27) is obtained by integrating $\Pi_{B} / \lambda$ with respect to $m^{2}$ in Eq. (A.7), and using the same value for the scale factor to remove the divergent terms in the $\overline{\mathrm{MS}}$ scheme.

The finite temperature part is given by the second term inside the square bracket of Eq. A.2. After integration over $\boldsymbol{p}_{\perp}$ and $p_{z}$, the function $\Pi_{T, B}$ can be written as 
in Eq. (28). Consequently, by integrating $\Pi_{T, B} / \lambda$ with respect to $m^{2}$ we get the function $\Omega_{T, B}$ in Eq. (28).

In the limit $T \ll m_{B}$, one can use the steepest descent approximation [39]. By scaling the variable $s \rightarrow s^{\prime} / m_{B} T$ in Eq. (28), both integrals can be expressed in terms of

$$
I=\int d s^{\prime} e^{\beta m_{B} f\left(s^{\prime}\right)} g\left(s^{\prime}\right) \approx \frac{\sqrt{2 \pi} g\left(s_{0}\right) e^{\beta m_{B} f\left(s_{0}\right)}}{\left|\beta m_{B} f^{\prime \prime}\left(s_{0}\right)\right|^{1 / 2}}
$$

with $f(s)=-\left(s+n^{2} / 4 s\right)$ and with the saddle point $s_{0}=n / 2$. With this approximation we arrive at Eqs. (29) and (35).
[1] G. Baym and E. Flowers, Nucl. Phys. A 222, 29 (1974); C. K. Au and G. Baym, Nucl. Phys. A 136, 500 (1974); C. K. Au and G. Baym, Phys. Lett. B 51, 1 (1974).

[2] N. K. Glendenning, Compact Stars: Nuclear Physics, Particle Physics and General Relativity (Springer-Verlag New York, 2000), 2nd ed.

[3] D. T. Son and M. A. Stephanov, Phys. Rev. Lett. 86, 592 (2001); Phys. At. Nucl. 64, 834 (2001).

[4] J. B. Kogut and D. Toublan, Phys. Rev. D 64, 034007 (2001).

[5] K. Splittdorff, D. Toublan, and J. I. M. Verbaarshot, Nucl. Phys. B 620, 290 (2002).

[6] T. Herpay and P. Kovacs, Phys. Rev. D 78, 116008 (2008).

[7] D. Ebert and K. G. Klimenko, Eur. Phys. J. C 46, 771 (2006).

[8] H. Abuki, R. Anglani, R. Gatto, M. Pellicoro and M. Ruggieri, Phys. Rev. D 79, 034032 (2009).

[9] J. O. Andersen and L. Kyllingstad, J. Phys. G 37, 015003 (2010).

[10] M. Loewe and C. Villavicencio, Phys. Rev. D 67, 074034 (2003); Phys. Rev. D 70, 074005 (2004).

[11] M. Loewe and C. Villavicencio Phys. Rev. D 71, 094001 (2005).

[12] D. T. Son and M. A. Stephanov, Phys. Rev. D 77014021 (2008).

[13] D. E. Kharzeev, L. D. McLerran and H. J. Warringa, Nucl. Phys. A 803, 227 (2008).

[14] A. J. Mizher, M. N. Chernodub and E. S. Fraga, Phys. Rev. D 82, 105016 (2010).

[15] A. J. Mizher and E. S. Fraga, Nucl. Phys. A 831, 91 (2009).

[16] E. S. Fraga and L. F. Palhares, Phys. Rev. D 86, 016008 (2012).

[17] M. R. Schafroth, Phys. Rev. 100, 463 (1955).

[18] R. M. May, J. Math. Phys. 6, 1462 (1965).

[19] J. Daicic, N. E. Frankel and V. Kowalenko, Phys. Rev. Lett. 71, 1779 (1993).

[20] P. Elmfors, P. Liljenberg, D. Pearson, B.-S. Skagerstam, Phys. Lett. B 348, 462 (1995).

[21] H. Perez-Rojas, Phys. Lett. B 379, 148 (1996).

[22] V. P. Gusynin, V. A. Miransky and I. A. Shovkovy, Phys.
Lett. B 349, 477 (1995); D.-S. Lee, C. N. Leung and Y. J. Ng, Phys. Rev. D 55, 6504 (1997).

[23] S. P. Klevansky and R. H. Lemmer, Phys. Rev. D 39 3478 (1989).

[24] K. G. Klimenko, Teoret. Mat. Fiz., 89, 211 (1991) [Theor. Math. Phys. 89, 1161 (1991)]; Teoret. Mat. Fiz., 90, 3 (1992) [Theor. Math. Phys. 90, 1 (1992)]; Z. Phys. C 54, 323 (1992).

[25] D. C. Duarte, R. L. S. Farias, and R. O. Ramos, Phys. Rev. D 84, 083525 (2011).

[26] B. J. Harrington and H. K. Shepard, Phys. Rev. D 16, 3437 (1977).

[27] P. E. de Brito and H. N. Nazareno, Eur. J. Phys. 28, 9 (2007).

[28] A. Ayala, A. Sanchez, G. Piccinelli and S. Sahu, Phys. Rev. D 71, 023004 (2005).

[29] A. Ayala, A. Bashir, A. Raya and A. Sanchez, Phys. Rev. D 80, 036005 (2009).

[30] J. Navarro, A. Sanchez, M. E. Tejeda-Yeomans, A. Ayala and G. Piccinelli, Phys. Rev. D 82, 123007 (2010).

[31] P. Fendley, Phys. Lett. B 196175 (1987).

[32] I. A. Shovkovy, arXiv:1207.5081.

[33] M. D'Elia, S. Mukherjee and F. Sanfilippo, Phys. Rev. D 82, 051501 (2010).

[34] M. D'Elia and F. Negro, Phys. Rev. D 83, 114028 (2011).

[35] E. -M. Ilgenfritz, M. Kalinowski, M. Müller-Preussker, B. Petersson and A. Schreiber, Phys. Rev. D 85, 114504 (2012).

[36] See, for example, N. Temme, Special Functions: An Introduction to the Classical Functions of Mathematical Physics, (Wiley-Interscience, New York, 1992).

[37] G. S. Bali, F. Bruckmann, G. Endrodi, Z. Fodor, S. D. Katz, S. Krieg, A. Schafer and K. K. Szabo, JHEP02, 044 (2012).

[38] G. S. Bali, F. Bruckmann, G. Endrodi, Z. Fodor, S. D. Katz and A. Schafer, arXiv:1206.4205 [Phys. Rev. D (to be published)].

[39] G. B. Arfken, H. J. Weber, and F. E. Harris, Mathematical Methods for Physicists (Academic Press, New York, 2005), 6th ed. 